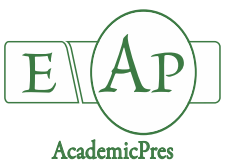

Fecowicz M et al. (2020)
Notulae Botanicae Horti Agrobotanici Cluj-Napoca 48(4):2032-2049
DOI:10.15835/48412148
Research Article

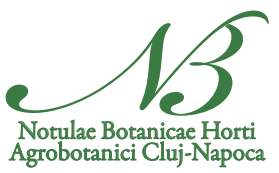

\title{
Allelopathic influence of medicinal plant Filipendula vulgaris Moench on germination process
}

\author{
Michał FECOWICZ ${ }^{1}$, Katarzyna MOŻDŻEŃ², \\ Beata BARABASZ-KRASNY², Alina STACHURSKA-SWAKOŃ ${ }^{1 *}$ \\ IJagiellonian University, Institute of Botany, Gronostajowa 3 St., 30-387 Kraków, Poland; michal.fecowicz@doctoral.uj.edu.pl; \\ alina.stachurska-swakon@uj.edu.pl (*corresponding author) \\ ${ }^{2}$ Pedagogical University of Krakow, Institute of Biology, Podchorązych 2 St., 30-084 Kraków, Poland; \\ katarzyna.mozdzen@up.krakow.pl; beata.barabasz-krasny@up.krakow.pl
}

\begin{abstract}
The allelopathic potential of medicinal plant Filipendula vulgaris Moench, originated from two natural habitats: calcareous grasslands and humid meadows, was investigated using radish (Raphanus sativus L. var. radicula Pers.) as a model plant. Suppression influence of aqueous extracts at concentrations: $1 \%, 5 \%, 10 \%$, obtained separately from under and aboveground parts of the species, was studied testing germination capacity, biomass and biometry of seedling, and electrolyte leakage. Additionally, two cultivars of the radish were used to test the sensitivity of frequently used plant in the laboratory tests. This study revealed the inhibiting effect of high concentration aqueous extracts from $F$. vulgaris organs on the germination of radish. It was significant for seed vigour index, speed emergence, germination stress tolerance index, and mean germination time. However, the stimulating effect of $1 \%$ of aqueous extracts from dropwort roots and shoots on the germination indexes was documented. Similarly, low concentration extracts had a positive effect on seedling elongation which was visible in biometric analyses and inhibition percentage index but it was not so obvious in the biomass analyses. In general, the aboveground extracts from dropwort grown on calcareous grassland demonstrated higher allelopathic potential than extracts from underground parts and meadow habitat.
\end{abstract}

Keywords: dropwort; electrolyte leakage; germination; medicinal plant; seeds

\section{Introduction}

Medicinal plants have a long history as a source of potential therapeutic agents, that have been incorporated into modern medicine (e.g. Kamboj, 2000; Tapsell et al., 2006; Verma and Singh, 2008; Atanasov et al., 2015). It is estimated that almost 53,000 flowering plant species are considered medicinal plants (Wakdidar, 2004). They are used mainly due to their natural compounds with biological activity that have a positive effect on maintaining human health. Additionally, secondary metabolites in medicinal plants have been reported as potent growth inhibitory agents indicating that such plants serve as a storehouse for allelopathic compounds (Batish et al., 2006). In the last decade, there is a growing interest to estimate the allelopathic potential of medicinal plants (e.g. Fujii et al., 2003; Amini et al., 2014; Skrzypek et al., 2016; Puła 
et al., 2020). Behind the researches of allelopathic activity is the purpose of better practice in the cultivation of medicinal plants as well as a future prospect in controlling major crop weeds for sustainable agricultural production (Islam et al., 2018). Increasing consumer awareness of herbicide residues in production practices leads to increased demands for organic products or safer foods (McErlich and Boydston, 2013; Tal, 2018).

Possibility of using plants compounds as natural pesticide promote sustainable agriculture and address audience concerns on the effects of pesticides on the environment and human health (Kropff and Walter, 2000; Dayan et al., 2009). There are suggestions that most allelopathic compounds will be biodegradable and also less polluting than traditional pesticides owing to their shorter half-lives (Daizy et al., 1995).

Filipendula vulgaris Moench (syn. F. hexapetala Gilib., Rosaceae), commonly known as dropwort (or fern-leaf dropwort), is one of the plant species used in traditional medicine. The use of the species in folk medicine was reported from Europe in countries such as Poland (Mowszowicz, 1985; Oszmiański et al., 2007), Romania (Imbrea et al., 2010), Russia (Olennikov and Kruglova, 2013) or Serbia (Tucakov, 1973). The entire plant or their parts (flowers, leaves, tubers) were used in case of breathlessness, wheezing, sore throats and congestion, stomachache and diarrhea, rheumatism (Mowszowicz, 1985). Published studies reveal antibacterial, anti-inflammatory, antipyretic, antihyperalgesic, and antioxidant properties, as well as nootropic activity (Maksimović et al., 2007; Pavlovic et al., 2007; Katanić et al., 2015; 2018; Shilova and Suslov, 2015; Shilova et al., 2015; Samardžić et al., 2016).

The plant contains active substances such as spiraeoside, phenolic acids (ellagic, gallic, caffeic, salicylic, syringic, chlorogenic, rosmarinic), flavonoids (quercetin, hyperoside, kaempferol, apigenin, luteolin, dihydroquercetin, isoquercitrin, avicularin, spiraeoside, rutin), tannins (rugosins, tellimagrandins), saponins, amino acids and other (Oszmianski et al., 2007; Bączek et al., 2011, 2012; Vengerovsky et al., 2011; Capecka et al., 2012; Olennikov and Kruglova, 2013; Movsumov et al., 2017, Shilova and Korotkova, 2017; Katanić et al., 2018).

Filipendula vulgaris is a rosette-forming perennial and clonal herb bearing short rhizomes and tubers (Klimešová and Klimeš, 2006). The flowering stalks are up to $80 \mathrm{~cm}$ high, consisting of numerous cream-white flowers, mainly pollinated by insects or wind (Clapham et al., 1987; Weidema et al., 2000). Filipendula vulgaris is classified as euro-siberian species (Zając and Zając, 2009) and occurs in north-western Africa through Europe and middle Asia range. Dropwort is included as diagnostic species for plant communites of Festuco-Brometea class, where calcareous grasslands belongs (Medwecka-Kornaś et al., 1966; Towpasz and Stachurska-Swakoń, 2011, 2012). Moreover, the species may also grow in moist meadows from the Caricetalia davalianae order and Molinietalia order (Dubiel et al., 1999; Towpasz and Stachurska-Swakoń, 2009). The studies focusing on biology of this species are scarce (Kostrakiewicz-Gierałt and Stachurska-Swakoń, 2017).

The aim of the study was to determine the allelopathic influence of aqueous extracts from Filipendula vulgaris on germination process and early growth on model plant Raphanus sativus $\mathrm{L}$. var. radicula Pers. We used separately underground and aboveground parts of dropwort originated from two natural habitats: humid meadow and calcareous grassland to examine the potential of allelopathy and the influence of habitat on production of allelopathic compounds. The germination capacity of seeds was tested by determining the germination indexes, the biometric analysis of seedlings was performed, the value of fresh and dry mass was determined and the percentage of destabilisation of cell membranes was measured. In the experiment two cultivars of radish were used to test the sensitivity of the model plant.

\section{Materials and Methods}

\section{Plant material}

Filipendula vulgaris specimens were collected from two natural habitats in southern Poland: humid meadows and calcareous grasslands in July 2019 (Figure 1). The humid meadows from the alliance Molinion caeruleae, Molinio-Arrhenatheretea class, were situated on the low flood terrace of the Vistula river where the 
limestone monadnocks (Jurassic-Cretaceous) and tectonic depressions of the Brama Krakowska gate occur $\left(50^{\circ} 01^{\prime} 48.8^{\prime \prime} \mathrm{N} 19^{\circ} 52^{\prime} 26.7^{\prime \prime} \mathrm{E}\right.$, Silesian-Krakow Upland). Histic Aerosol is reported as the soil unit for the place where the meadows occurred (Skiba et al., 2010). The calcareous grasslands, represented Thalictro-Salvietum association from Festuco-Brometea class, were located in the Miechów Upland (50¹8'59.1"N 200.1'14.3"E, Małopolska Upland). Rendzic and calcareous leptosols are the soil units that prevailed in the area.

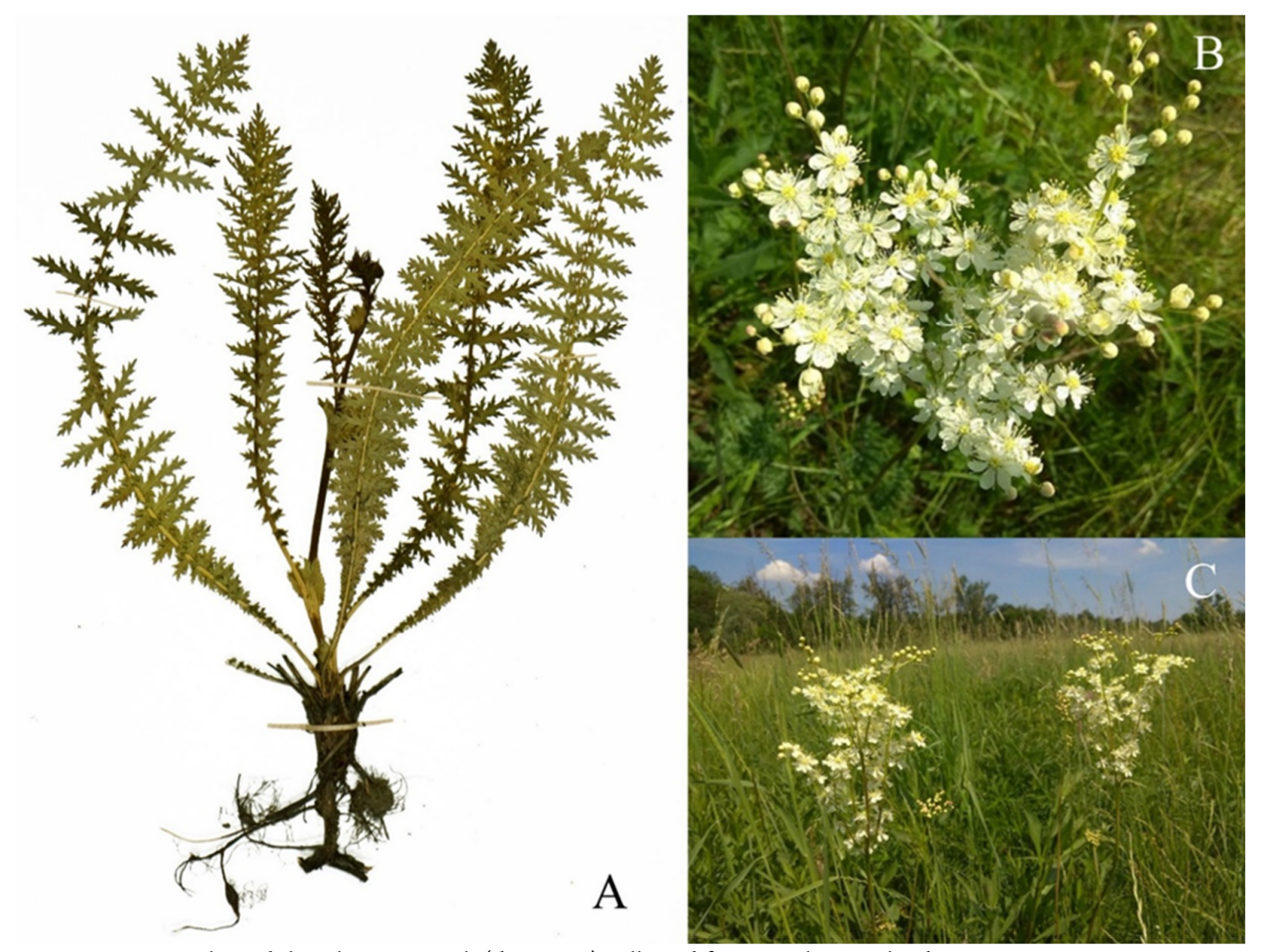

Figure 1. Filipendula vulgaris Moench (dropwort) collected from southern Poland

A - before flowering with a rosette of leaves, rhizomes, and tubers, B - inflorescence, C - specimens in meadow habitat (Photo M. Fecowicz)

Fresh material of the species was selected in the laboratory to eliminate defective parts (e.g. damaged, infected leaves, roots). Then the plants were separated into underground parts (roots, tubers, rhizomes) and aboveground parts (shoots, leaves). In the text, the term "roots" is used for underground parts and "shoots" for aboveground parts of the species. The plant material was dried at the temperature of $25^{\circ} \mathrm{C}$, in the dark for 3 weeks in a dryer (Wamed SUP-100, Poland). The dry plant material of $F$. vulgaris was stored in the paper bags, in the dark, in a desiccator to avoid microbiological destruction of allelopathic compounds present in it.

Radish Raphanus sativus L. var. radicula Pers was selected for the study as a model organism due to the short life cycle and low environmental requirements for germination. Two commonly cultivars of radish: 'Rowa' and 'Krakowianka' were used in the experiment. Both cultivars require sunny areas, rich and permeable soil with low nitrogen content. They differ in the length of the growing season: 'Rowa': 28-32 days, 'Krakowianka': 33-37 days (Kupczak, 2011). The seeds of radish cultivars were purchased from POLAN Sp.z.o.o. Breeding and Seed Horticulture in Kraków (Poland). 


\section{Preparation of aqueous extract solutions}

Dry plant material was ground separately for under and aboveground parts of dropwort in a mill (Braun, 4045 type, Germany) and used to prepare the aqueous extracts of various concentrations: $1 \%, 5 \%$, and $10 \%$. Each extract, depending on the concentration, was flooded with a suitable amount of distilled water (e.g. $5 \%=$ $5 \mathrm{~g}$ plant material $+95 \mathrm{ml}$ distilled water, $10 \%=10 \mathrm{~g}$ plant material $+90 \mathrm{ml}$ distilled water) and left for 24 hours, at $25^{\circ} \mathrm{C}$ temperature, in the dark to extract the compounds. The extracts obtained were filtered through a filter paper on a Büchner funnel by Whatman (Grade 1: $11 \mu \mathrm{m}$ - medium flow filter paper) filter paper, using a vacuum pump (PL2/3, Al-Conbest, USA), and then stored in a refrigerator for the duration of the experiment.

\section{Seed germination conditions}

Radish seeds were sterilised in $1 \%$ sodium hypochlorite for $10 \mathrm{~min}$, then rinsed with distilled water (3x). In the experiment, seeds were put on the sterile Petri dishes ( 25 seeds per dish) with 3 layers of filter paper (Whatman, Grade 1: $11 \mu \mathrm{m}$ - medium flow filter paper) soaked with $5 \mathrm{ml}$ the aqueous extract of dropwort: separately with different concentrations from under and aboveground parts. Then seeds on dishes were placed in a growth chamber (Angelantoni Industrie, Italy) at $25^{\circ} \mathrm{C} / 20^{\circ} \mathrm{C}$, photoperiod $200 \mu \mathrm{mol} \cdot \mathrm{m}^{-2} \cdot \mathrm{s}^{-1}(12 \mathrm{~h}$ day / $12 \mathrm{~h}$ night), and relative air humidity (RH) $80-90 \%$. The control group was radish seeds treated with distilled water in the same volumes as extract watered seeds. During the experiment, the number of germinated seeds was checked every $24 \mathrm{~h}$. Those that had a hypocotyl at least $2 \mathrm{~mm}$ in length were considered germinated. After 7 days, the effect of $F$. vulgaris aqueous extracts on germination and early growth of radish was measured.

\section{Germination indexes}

To check the influence of aqueous extract on the germination process the following indexes were used: seedling vigour index (SVI), speed of emergence (SE) (Mazur, 2020), rate of germination (RG). Mean germination time (MGT) and germination stress tolerance index (GSTI) were calculated according to the formulas used by Możdżeń et al. (2020).

SVI $=[$ seedling length $(\mathrm{cm}) \times$ percentage of germinated seeds $] / 100$

$\mathrm{SE}=[$ number of germinated seeds on the first day of germination / number of germinated seeds on the last day of germination $] \times 100$

$\mathrm{RG}=[(\mathrm{n} 1+\mathrm{n} 2+\mathrm{nn}) /(\mathrm{n} 1 \times \mathrm{T} 1)+(\mathrm{n} 2 \times \mathrm{T} 2)+(\mathrm{n} 3 \times \mathrm{T} 3)+\ldots)] \times 100$

where: $\mathrm{n} 1=$ number of germinated seeds on time $\mathrm{T} 1 ; \mathrm{n} 2=$ number of germinated seeds on time $\mathrm{T} 2 ; \mathrm{n} 3$ $=$ number of germinated seeds on timeT3

$\mathrm{MGT}=\Sigma \mathrm{fx} / \Sigma \mathrm{f}$

where: $x$ is the number of new seeds germinating on day $f$ and $f$ is the number of days from the beginning GSTI $(\%)=[$ PI of stressed seeds $/$ PI of control seeds $] \times 100$

where: PI is a speed of germination index calculated according to the formula: $\mathrm{PI}=\mathrm{nd} 2 \times(1.00)+\mathrm{nd} 4$ $\times(0.75)+\mathrm{nd} 6 \times(0.50)+\mathrm{nd} 8 \times(0.25) ; \mathrm{nd} 2$, nd $4, \mathrm{nd} 6$ and $\mathrm{nd} 8$ are the seed numbers, germinating on days 2 , 4,6 and 8 , respectively.

\section{Seedlings elongation}

The length of roots and hypocotyls, as well as whole seedlings, was measured using a caliper (Topex 31C615, Poland), with an accuracy of $1 \mathrm{~mm}$. Besides, the seedling length was expressed as a percentage of control by using an inhibition percentage (IP) index:

$\mathrm{IP}=\left[1-\left(\mathrm{L}_{\mathrm{E}} / \mathrm{L}_{\mathrm{C}}\right)\right] \times 100$

where: IP - growth inhibition index (\%), LE - plant length $(\mathrm{cm})$ treated with aqueous extract, LC plant length $(\mathrm{cm})$ from the control. 
Inhibition percentage (IP) of growth (as control \%) - a minus (-) value indicates growth stimulation, and a plus $(+)$ value indicates growth inhibition.

Fresh and dry masses, water content

Fresh mass (g) of single seedlings was weighted (Ohaus Adventurer Pro, USA). Dry mass of seedlings was determined after $48 \mathrm{~h}$ of drying the fresh material in a dryer (Wamed SUP-100, Poland) at temperature $105^{\circ} \mathrm{C}$. Additionally, the percentage of dry mass and total water content were determined.

Dry mass $(\%)=($ dry mass $/$ fresh mass $) \times 100$

$\mathrm{H}_{2} \mathrm{O}(\%)=[($ fresh mass - dry mass $) /$ fresh mass $] \times 100$

\section{Electrolyte leakage}

The percentage of destabilisation of cell membranes of radish seedlings was determined by slightly modifying the method described in the study of Możdżeń et al. (2020). A single radish seedling was placed in polypropylene falcons with $30 \mathrm{ml}$ distilled water. Each of the samples was shaken for $3 \mathrm{~h}$ on a shaker (Labnet, Rocker, USA) to measure the electrolytes leakage from living seedlings (EL). Electrolyte leakage was measured by conductometer (CX-701, Elmetron, Poland) with electrode $(\mathrm{K}=1.02)$ (Elmetron, Poland). The seedlings in distilled water were frozen at $-75^{\circ} \mathrm{C}$ for $24 \mathrm{~h}$. The next day, samples were defrosted and shaken for $3 \mathrm{~h}$ to determine the total electrolyte leakage from dead seedlings (ED). The total percentage of electrolyte leakage was determined according to the formula:

$\mathrm{EL}=(\mathrm{EL} / \mathrm{ED}) \times 100$

\section{Statistical analysis}

The experiment was performed in three repetitions. The obtained results were statistically tested between repetitions of the experiment $(n=3, \pm S E)$ as well as between cultivars, habitats extracts, and organ extracts using the Duncan test at $\mathrm{p}<0.05$ with Statistica software.

\section{Results}

\section{Germination indexes}

The percentage of seeds germinated was the highest in the control. A decrease in seed germination capacity was observed with the increase in the concentration of aqueous extracts. The smallest differences were found between the control and 1\% of Filipendula vulgaris root and shoot extracts regardless of the habitats. The lowest number of germinated radish seeds was found on the $10 \%$ dropwort extracts. More germinated seeds were observed on root extracts than on shoots (Figure 2).

The seed vigour index (SVI) reached the significantly highest values for the investigated radish cultivars watered with the $1 \%$ aqueous extracts of $F$. vulgaris roots, compared to the control. In the case of extracts from shoots, the highest values of this parameter were recorded for the 'Krakowianka' cultivar treated with $1 \%$ and $5 \%$ of the extracts of plants growing on calcareous grassland. Regardless of the radish cultivar and the extract type, the lowest SVI values were found for seeds germinated on the 10\% extracts (Table 1).

Speed emergence (SE) values for radish seeds watered with root extracts, both from meadows and calcareous grasslands, were similar to the control at each concentration. The exception was $10 \%$ calcareous grassland root extracts, which significantly limited the SE. Aqueous extracts from shoots with a concentration of $1 \%$ did not affect the values of this parameter. Compared to the control samples, $5 \%$ and $10 \%$ extracts, especially from shoots obtained from calcareous grasslands, inhibited SE.

In comparison with the control, the only significant differences in the values of the rate of germination (RG) index were demonstrated for the 'Krakowianka' cultivar watered during germination with $10 \%$ shoot 
extracts from calcareous grassland. In other cases, in the values of this index, no statistically significant differences were observed.

Mean germination time (MGT) reached similar values between the control and $1 \% F$. vulgaris root and shoot extracts from both meadow and calcareous grassland. In the 'Rowa' cultivar, a lower value of this index was revealed for seeds treated, regardless of the concentration of the extract, with dropwort plant root extracts from the meadow. For the 'Krakowianka', significant differences were found only for seeds watered with 10\% extracts.
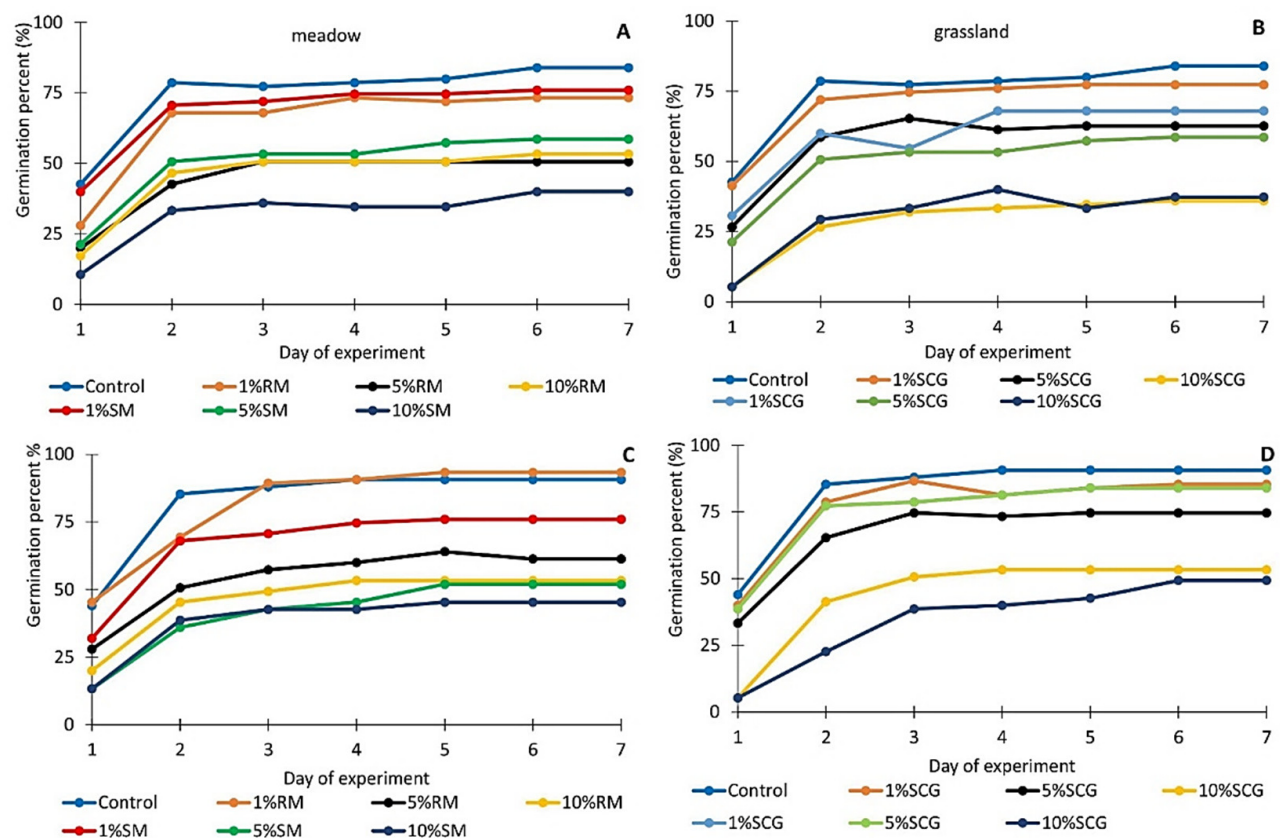

Figure 2. Germination capacity of Raphanus sativus L. var. radicula Pers. cv under influence of Filipendula vulgaris extracts

(A, B) - 'Rowa', (C, D) - 'Krakowianka' in seedlings 'watered with aqueous extracts from root and shoot Filipendula vulgaris Moench in different concentrations; Control - distilled water, 1, 5, 10RM - 1\%, 5\%, 10\% extract from root plant grown on meadow, 1, 5, 10RCG - 1\%, 5\%, 10\% extract from root plant grown on calcareous grasslands, 1, 5, $10 \mathrm{SM}-1 \%, 5 \%, 10 \%$ extract from shoot plant grown on meadow, 1, 5, 10SCG - 1\%, 5\%, 10\% extract from shoot plant grown on calcareous grasslands

In the remaining cases, irrespective of the cultivar, significant differences were observed between the control and extracts of $10 \%$ root extracts from calcareous grasslands and $5 \%$ and $10 \%$ shoot extracts from plants grown on meadows and calcareous grasslands.

Germination stress tolerance index (GST) achieved significantly the highest values for two radish cultivars watered with $1 \%$ extracts of plant shoots harvested from calcareous grasslands. Additionally, for the 'Rowa', a significant increase in the GST value was demonstrated for seeds watered with $1 \%$ extracts from the roots of plants growing on calcareous grasslands and shoots collected from meadows. The lowest values of this index were observed for 'Rowa' seeds watered with 10\% root extract and for 'Krakowianka' treated with 10\% shoot extracts obtained from calcareous grasslands (Table 1 ). 
Table 1. Germination parameters of Raphanus sativus L. var. radicula Pers. cv (A) - 'Rowa', (B) -

'Krakowianka' in seedlings 'watered with aqueous extracts from root and shoot Filipendula vulgaris

Moench in different concentrations

\begin{tabular}{|c|c|c|c|c|c|c|c|c|c|c|}
\hline \multirow{2}{*}{$\begin{array}{c}\text { Aqueous } \\
\text { extracts } \\
(\%)\end{array}$} & \multicolumn{2}{|c|}{ SVI } & \multicolumn{2}{|c|}{ SE } & \multicolumn{2}{|c|}{ RG } & \multicolumn{2}{|c|}{ MGT } & \multicolumn{2}{|c|}{ GST } \\
\hline & A & B & A & B & A & B & A & B & A & B \\
\hline Control & $\begin{array}{l}6.52 \mathrm{~b} \\
\pm 0.02 \\
\end{array}$ & $\begin{array}{l}6.94 \mathrm{~b} \\
\pm 0.02 \\
\end{array}$ & $\begin{array}{c}50.88 \mathrm{a} \\
\pm 2.12\end{array}$ & $\begin{array}{l}49.92 \mathrm{a} \\
\pm 2.00\end{array}$ & $\begin{array}{c}23.47 \mathrm{a} \\
\pm 1.12\end{array}$ & $\begin{array}{c}23.49 \mathrm{a} \\
\pm 1.02\end{array}$ & $\begin{array}{l}0.51 \mathrm{a} \\
\pm 0.02\end{array}$ & $\begin{array}{l}0.49 \mathrm{a} \\
\pm 0.01\end{array}$ & $\begin{array}{l}1.00 \mathrm{~b} \\
\pm 0.01\end{array}$ & $\begin{array}{l}1.00 \mathrm{~b} \\
\pm 0.01\end{array}$ \\
\hline $1 \mathrm{RM}$ & $\begin{array}{l}9.55 \mathrm{a} \\
\pm 0.05\end{array}$ & $\begin{array}{c}10.78 \mathrm{a} \\
\pm 0.05\end{array}$ & $\begin{array}{c}37.38 \mathrm{ab} \\
\pm 1.56\end{array}$ & $\begin{array}{c}48.43 \mathrm{a} \\
\pm 1.82\end{array}$ & $\begin{array}{c}23.10 \mathrm{a} \\
\pm 1.40\end{array}$ & $\begin{array}{c}23.04 \mathrm{a} \\
\pm 1.00\end{array}$ & $\begin{array}{l}0.38 \mathrm{~b} \\
\pm 0.01\end{array}$ & $\begin{array}{l}0.49 \mathrm{a} \\
\pm 0.01\end{array}$ & $\begin{array}{l}0.89 \mathrm{~b} \\
\pm 0.02\end{array}$ & $\begin{array}{l}0.94 \mathrm{~b} \\
\pm 0.01\end{array}$ \\
\hline $5 \mathrm{RM}$ & $\begin{array}{l}4.19 \mathrm{~d} \\
\pm 0.01 \\
\end{array}$ & $\begin{array}{l}7.15 \mathrm{~b} \\
\pm 0.04 \\
\end{array}$ & $\begin{array}{c}37.25 \mathrm{ab} \\
\pm 1.55 \\
\end{array}$ & $\begin{array}{c}45.37 \mathrm{a} \\
\pm 1.79 \\
\end{array}$ & $\begin{array}{c}22.98 \mathrm{a} \\
\pm 0.99 \\
\end{array}$ & $\begin{array}{c}23.03 \mathrm{a} \\
\pm 1.11 \\
\end{array}$ & $\begin{array}{l}0.39 \mathrm{~b} \\
\pm 0.01 \\
\end{array}$ & $\begin{array}{l}0.46 \mathrm{a} \\
\pm 0.01 \\
\end{array}$ & $\begin{array}{c}0.67 \mathrm{~cd} \\
\pm 0.01 \\
\end{array}$ & $\begin{array}{c}0.68 \mathrm{~cd} \\
\pm 0.01 \\
\end{array}$ \\
\hline $10 \mathrm{RM}$ & $\begin{array}{l}4.99 \mathrm{c} \\
\pm 0.01\end{array}$ & $\begin{array}{l}5.25 \mathrm{c} \\
\pm 0.01\end{array}$ & $\begin{array}{c}32.14 \mathrm{ab} \\
\pm 1.46\end{array}$ & $\begin{array}{c}37.78 \mathrm{ab} \\
\pm 1.52\end{array}$ & $\begin{array}{c}22.81 \mathrm{a} \\
\pm 1.12\end{array}$ & $\begin{array}{c}22.92 \mathrm{a} \\
\pm 1.14\end{array}$ & $\begin{array}{l}0.33 \mathrm{~b} \\
\pm 0.01\end{array}$ & $\begin{array}{l}0.38 \mathrm{~b} \\
\pm 0.01\end{array}$ & $\begin{array}{l}1.05 \mathrm{~b} \\
\pm 0.03\end{array}$ & $\begin{array}{c}0.88 \mathrm{bc} \\
\pm 0.01\end{array}$ \\
\hline $1 \mathrm{RCG}$ & $\begin{array}{c}10.52 \mathrm{a} \\
\pm 0.05\end{array}$ & $\begin{array}{c}11.14 \mathrm{a} \\
\pm 0.05\end{array}$ & $\begin{array}{c}53.88 \mathrm{a} \\
\pm 2.15\end{array}$ & $\begin{array}{c}46.42 \mathrm{a} \\
\pm 1.92 \\
\end{array}$ & $\begin{array}{c}23.51 \mathrm{a} \\
\pm 1.10 \\
\end{array}$ & $\begin{array}{c}23.41 \mathrm{a} \\
\pm 1.10 \\
\end{array}$ & $\begin{array}{l}0.53 \mathrm{a} \\
\pm 0.02 \\
\end{array}$ & $\begin{array}{l}0.47 \mathrm{a} \\
\pm 0.01 \\
\end{array}$ & $\begin{array}{l}1.50 \mathrm{a} \\
\pm 0.04 \\
\end{array}$ & $\begin{array}{l}1.63 \mathrm{~b} \\
\pm 0.05 \\
\end{array}$ \\
\hline $5 \mathrm{RCG}$ & $\begin{array}{l}6.79 \mathrm{~b} \\
\pm 0.02\end{array}$ & $\begin{array}{l}7.55 \mathrm{~b} \\
\pm 0.04 \\
\end{array}$ & $\begin{array}{c}42.86 \mathrm{a} \\
\pm 1.92 \\
\end{array}$ & $\begin{array}{l}44.22 \mathrm{a} \\
\pm 1.56\end{array}$ & $\begin{array}{c}22.89 \mathrm{a} \\
\pm 1.10\end{array}$ & $\begin{array}{c}23.22 \mathrm{a} \\
\pm 1.12\end{array}$ & $\begin{array}{l}0.43 \mathrm{a} \\
\pm 0.01 \\
\end{array}$ & $\begin{array}{l}0.45 \mathrm{a} \\
\pm 0.01 \\
\end{array}$ & $\begin{array}{l}0.81 \mathrm{~b} \\
\pm 0.02 \\
\end{array}$ & $\begin{array}{c}0.87 \mathrm{bc} \\
\pm 0.02\end{array}$ \\
\hline 10RCG & $\begin{array}{l}2.84 \mathrm{ef} \\
\pm 0.01\end{array}$ & $\begin{array}{l}4.23 \mathrm{~d} \\
\pm 0.01\end{array}$ & $\begin{array}{c}14.07 \mathrm{c} \\
\pm 1.02\end{array}$ & $\begin{array}{c}9.21 \mathrm{~cd} \\
\pm 1.00\end{array}$ & $\begin{array}{c}22.36 \mathrm{a} \\
\pm 1.15\end{array}$ & $\begin{array}{c}21.93 \mathrm{ab} \\
\pm 1.52 \\
\end{array}$ & $\begin{array}{c}0.15 \mathrm{~cd} \\
\pm 0.01\end{array}$ & $\begin{array}{l}0.10 \mathrm{~d} \\
\pm 0.05\end{array}$ & $\begin{array}{l}0.52 \mathrm{~d} \\
\pm 0.01\end{array}$ & $\begin{array}{c}0.69 \mathrm{~cd} \\
\pm 0.01\end{array}$ \\
\hline $1 S M$ & $\begin{array}{l}7.46 \mathrm{~b} \\
\pm 0.04 \\
\end{array}$ & $\begin{array}{l}5.03 \mathrm{c} \\
\pm 0.01 \\
\end{array}$ & $\begin{array}{c}52.63 \mathrm{a} \\
\pm 2.00 \\
\end{array}$ & $\begin{array}{l}42.33 \mathrm{a} \\
\pm 1.62 \\
\end{array}$ & $\begin{array}{c}23.56 \mathrm{a} \\
\pm 1.10 \\
\end{array}$ & $\begin{array}{c}23.14 \mathrm{a} \\
\pm 1.00 \\
\end{array}$ & $\begin{array}{l}0.53 \mathrm{a} \\
\pm 0.01 \\
\end{array}$ & $\begin{array}{c}0.42 \mathrm{ab} \\
\pm 0.01 \\
\end{array}$ & $\begin{array}{r}2.33 \mathrm{a} \\
\pm 0.06 \\
\end{array}$ & $\begin{array}{l}1.49 \mathrm{~b} \\
\pm 0.05 \\
\end{array}$ \\
\hline $5 \mathrm{SM}$ & $\begin{array}{l}7.76 \mathrm{~b} \\
\pm 0.04\end{array}$ & $\begin{array}{l}5.31 \mathrm{c} \\
\pm 0.01 \\
\end{array}$ & $\begin{array}{c}37.25 \mathrm{ab} \\
\pm 1.44 \\
\end{array}$ & $\begin{array}{c}26.03 \mathrm{~b} \\
\pm 1.02 \\
\end{array}$ & $\begin{array}{c}23.35 \mathrm{a} \\
\pm 1.12 \\
\end{array}$ & $\begin{array}{c}22.10 \mathrm{a} \\
\pm 0.99 \\
\end{array}$ & $\begin{array}{l}0.36 \mathrm{~b} \\
\pm 0.01 \\
\end{array}$ & $\begin{array}{l}0.26 \mathrm{c} \\
\pm 0.01 \\
\end{array}$ & $\begin{array}{l}0.73 \mathrm{c} \\
\pm 0.01\end{array}$ & $\begin{array}{l}0.60 \mathrm{~d} \\
\pm 0.01\end{array}$ \\
\hline $10 S M$ & $\begin{array}{l}3.06 \mathrm{e} \\
\pm 0.01 \\
\end{array}$ & $\begin{array}{l}4.06 \mathrm{~d} \\
\pm 0.01 \\
\end{array}$ & $\begin{array}{c}22.29 \mathrm{~b} \\
\pm 1.38 \\
\end{array}$ & $\begin{array}{c}32.96 \mathrm{ab} \\
\pm 1.52 \\
\end{array}$ & $\begin{array}{c}21.80 \mathrm{ab} \\
\pm 1.01 \\
\end{array}$ & $\begin{array}{c}22.83 \mathrm{a} \\
\pm 1.00 \\
\end{array}$ & $\begin{array}{l}0.27 \mathrm{c} \\
\pm 0.01 \\
\end{array}$ & $\begin{array}{l}0.29 \mathrm{c} \\
\pm 0.01 \\
\end{array}$ & $\begin{array}{c}0.66 \mathrm{~cd} \\
\pm 0.01 \\
\end{array}$ & $\begin{array}{l}0.96 \mathrm{~b} \\
\pm 0.01 \\
\end{array}$ \\
\hline 1SCG & $\begin{array}{l}7.84 \mathrm{~b} \\
\pm 0.04 \\
\end{array}$ & $\begin{array}{c}10.06 \mathrm{a} \\
\pm 0.05 \\
\end{array}$ & $\begin{array}{c}44.13 \mathrm{a} \\
\pm 1.73 \\
\end{array}$ & $\begin{array}{c}45.58 \mathrm{a} \\
\pm 2.10 \\
\end{array}$ & $\begin{array}{c}23.08 \mathrm{a} \\
\pm 0.89 \\
\end{array}$ & $\begin{array}{c}23.27 \mathrm{a} \\
\pm 1.22 \\
\end{array}$ & $\begin{array}{l}0.45 \mathrm{a} \\
\pm 0.01 \\
\end{array}$ & $\begin{array}{l}0.46 \mathrm{a} \\
\pm 0.01 \\
\end{array}$ & $\begin{array}{l}1.81 \mathrm{a} \\
\pm 0.07 \\
\end{array}$ & $\begin{array}{r}1.92 \mathrm{a} \\
\pm 0.08 \\
\end{array}$ \\
\hline 5SCG & $\begin{array}{l}6.49 \mathrm{~b} \\
\pm 0.02 \\
\end{array}$ & $\begin{array}{l}9.98 \mathrm{a} \\
\pm 0.05 \\
\end{array}$ & $\begin{array}{c}22.46 \mathrm{~b} \\
\pm 1.11 \\
\end{array}$ & $\begin{array}{c}16.68 \mathrm{~b} \\
\pm 1.12 \\
\end{array}$ & $\begin{array}{c}22.48 \mathrm{a} \\
\pm 1.00 \\
\end{array}$ & $\begin{array}{c}21.80 \mathrm{ab} \\
\pm 1.02 \\
\end{array}$ & $\begin{array}{l}0.36 \mathrm{~b} \\
\pm 0.01 \\
\end{array}$ & $\begin{array}{r}0.46 \mathrm{a} \\
\pm 0.01 \\
\end{array}$ & $\begin{array}{l}0.83 \mathrm{~b} \\
\pm 0.01 \\
\end{array}$ & $\begin{array}{l}1.00 \mathrm{~b} \\
\pm 0.01 \\
\end{array}$ \\
\hline 10SCG & $\begin{array}{l}4.08 \mathrm{~d} \\
\pm 0.01\end{array}$ & $\begin{array}{l}5.15 \mathrm{c} \\
\pm 0.01 \\
\end{array}$ & $\begin{array}{c}14.39 \mathrm{~b} \\
\pm 1.04 \\
\end{array}$ & $\begin{array}{c}11.61 \mathrm{bc} \\
\pm 1.01\end{array}$ & $\begin{array}{c}22.26 \mathrm{a} \\
\pm 1.12 \\
\end{array}$ & $\begin{array}{c}21.02 \mathrm{~b} \\
\pm 1.00\end{array}$ & $\begin{array}{c}0.14 \mathrm{~cd} \\
\pm 0.01\end{array}$ & $\begin{array}{l}0.11 \mathrm{~d} \\
\pm 0.01 \\
\end{array}$ & $\begin{array}{c}0.65 \mathrm{~cd} \\
\pm 0.01\end{array}$ & $\begin{array}{l}0.45 \mathrm{e} \\
\pm 0.01 \\
\end{array}$ \\
\hline
\end{tabular}

Control - distilled water, $1,5,10 \mathrm{RM}-1 \%, 5 \%, 10 \%$ extract from root plant grown on meadow, 1, 5, 10RCG - 1\%, $5 \%, 10 \%$ extract from root plant grown on calcareous grasslands, 1, 5, 10SM - 1\%, 5\%, 10\% extract from shoot plant grown on meadow, $1,5,10 \mathrm{SCG}-1 \%, 5 \%, 10 \%$ extract from shoot plant grown on calcareous grasslands; mean values $(\mathrm{n}=5, \pm \mathrm{SE})$ marked different letter differ statistically; SVI - seedling vigour index, SE - speed of emergence, RG rate of germination, MGT - mean germination time, GSTI - germination stress tolerance index; mean values $(\mathrm{n}=5$,

\pm SE) marked different letter differ statistically (in column) according to Duncan test at $\mathrm{p}<0.05$

\section{Seedling elongation}

Regardless of the cultivar, $1 \%$ aqueous extracts from the F. vulgaris roots stimulated the length of seedlings' roots.

In the case of plant shoots collected from meadows, the elongation of this radish organ was decreased. The $10 \%$ extracts from roots obtained from calcareous grasslands and meadows also had a negative impact on root length. For seedlings watered with $5 \%$ extracts from shoots collected on calcareous grasslands, significant root elongation was observed (Figure $3 \mathrm{~A}$ ).

The aqueous extracts from the roots and shoots of dropwort had a positive effect on the elongation of the hypocotyl of radish seedlings, 'Rowa' and 'Krakowianka' cultivars, concerning the control. Generally, hypocotyl growth stimulation was observed with increasing concentrations (Figure 4A). 

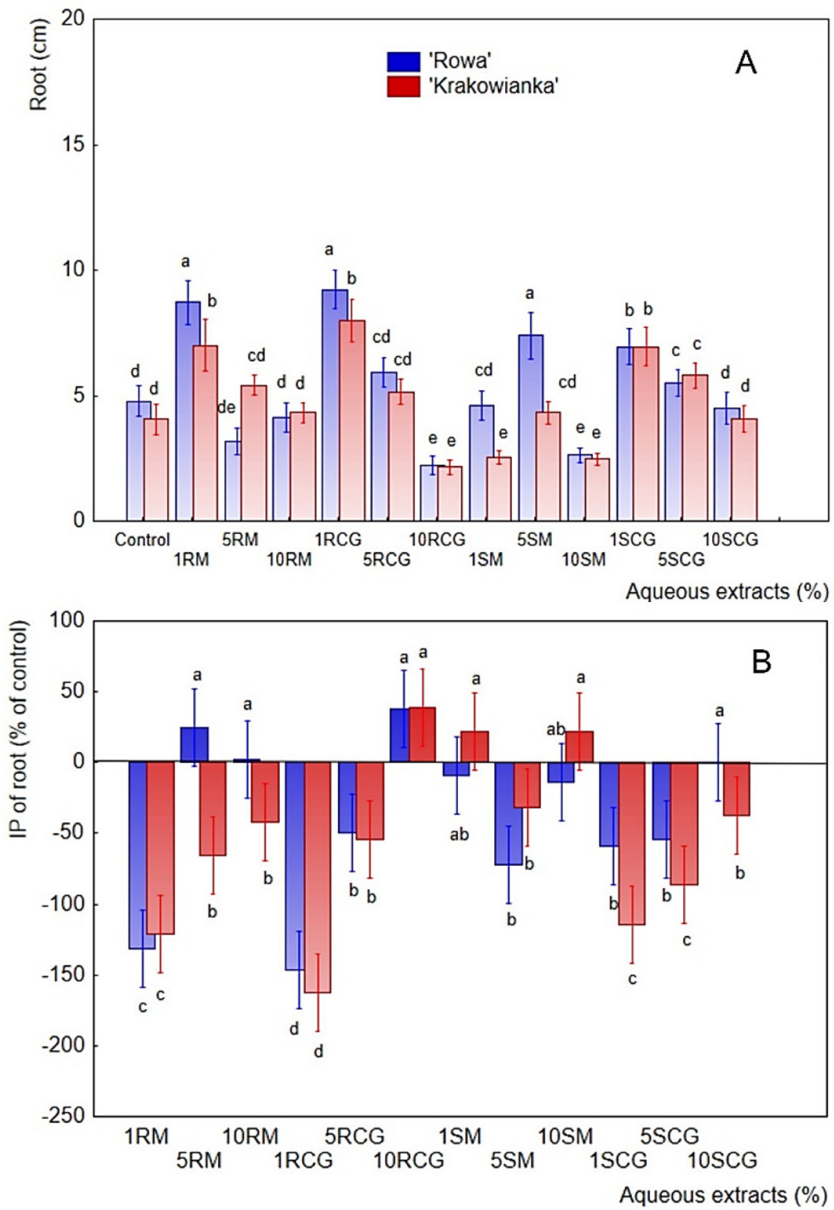

Figure 3. A - Length of roots of Raphanus sativus L. var. radicula Pers. 'Rowa' and 'Krakowianka' after 7 days of watering with from roots and shoots Filipendula vulgaris Moench with different concentrations (1, $5,10 \%$ ) and under control conditions; B - Inhibition percentage (IP) of growth (as control \%)

The horizontal line is the border with negative values that indicate elongation stimulation and positive values that indicate the percentage of inhibition of seedling growth; RM - extract from root plant grown on the meadow, SCG extract from shoot plant grown on calcareous grasslands; mean values $(\mathrm{n}=3, \pm \mathrm{SE})$ marked different letter differ statistically according to Duncan test at $\mathrm{p}<0.05$

Biometric analysis of whole seedlings revealed a stimulating effect for both radish cultivars, extracts from $1 \%$ of roots and $5 \%$ of shoots. Besides, a positive effect of shoot extracts at concentrations of $1 \%$ and $5 \%$ from calcareous grasslands was observed. Compared to the control, $5 \%$ root extracts of calcareous grassland plants also showed stimulation of seedling elongation (Figure 5A).

Morphometric analysis of the under- and aboveground organs of radish seedlings by determining the IP index as a percentage of control confirmed the positive and negative effect of extracts from $F$. vulgaris on the roots, hypocotyls, and whole radish seedlings in two cultivars. For this index, positive values indicate a higher percent growth inhibition. On the other hand, negative values show the positive influence of the extracts on organ elongation. The root length was inhibited by extracts with $10 \%$ concentrations, regardless of the dropwort organs. Contrary to the $1 \%$ extracts, which stimulated the growth of radish seedlings (Figure 3B). Elongation of the hypocotyls was stimulated relative to the control (Figure 4B). The length of the whole seedlings was generally stimulated, but to the highest extent by $1 \%$ dropwort extracts (Figure $5 \mathrm{~B}$ ). The smallest effects were found for extracts from shoots from calcareous grasslands (Figures 3-5). 

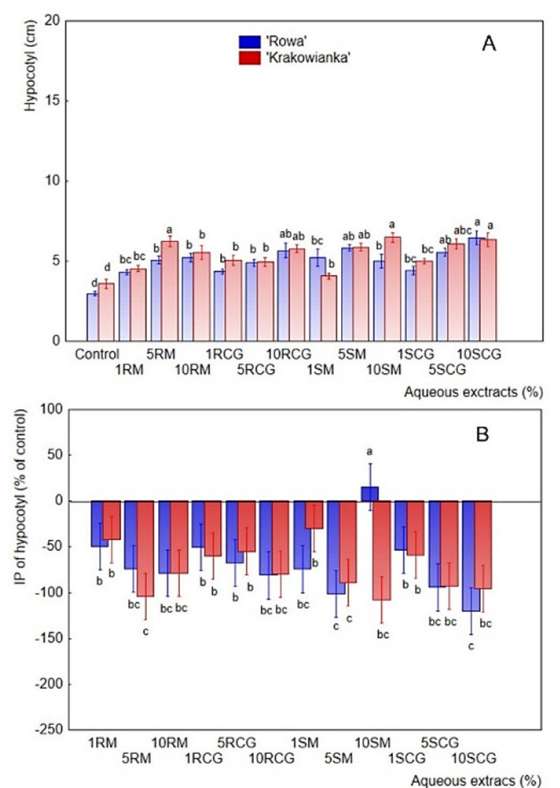

Figure 4. A - Length of hypocotyls of Raphanus sativus L. var. radicula Pers. 'Rowa' and 'Krakowianka' after 7 days of watering with aqueous extracts from roots and shoots Filipendula vulgaris Moench with different concentrations $(1,5,10 \%)$ and under control conditions; B - Inhibition percentage (IP) of growth (as control \%)

The horizontal line is the border with negative values that indicate elongation stimulation and positive values that indicate the percentage of inhibition of seedling growth; RM - extract from root plant grown on the meadow, SCG extract from shoot plant grown on calcareous grasslands; mean values $(n=3, \pm S E)$ marked different letter differ statistically according to Duncan test at $\mathrm{p}<0.05$
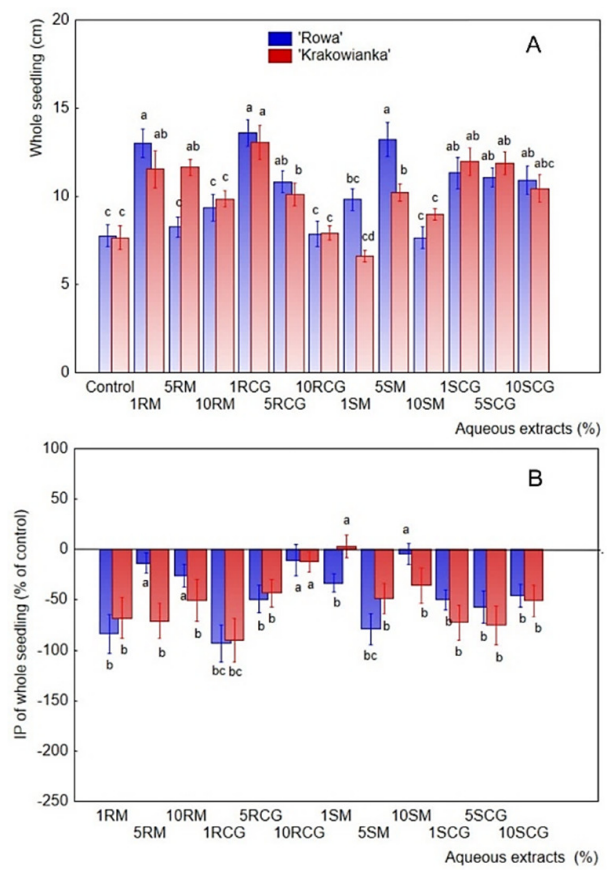

Figure 5. A - Length of whole seedlings of Raphanus sativus L. var. radicula Pers. 'Rowa' and 'Krakowianka' after 7 days of watering with aqueous extracts from roots and shoots Filipendula vulgaris Moench with different concentrations $(1,5,10 \%)$ and under control conditions; B - Inhibition percentage (IP) of growth (as control \%) 
The horizontal line is the border with negative values that indicate elongation stimulation and positive values that indicate the percentage of inhibition of seedling growth; RM - extract from root plant grown on the meadow, SCG extract from shoot plant grown on calcareous grasslands; mean values $(n=3, \pm S E)$ marked different letter differ statistically according to Duncan test at $\mathrm{p}<0.05$

Table 2. Fresh and dry mass and water content in seedlings Raphanus sativus L. var. radicula Pers. cv 'Rowa', 'Krakowianka' treatment the aqueous extracts from root and shoot Filipendula vulgaris Moench in different concentrations

\begin{tabular}{|c|c|c|c|c|c|c|c|c|c|c|c|c|c|}
\hline \multirow{2}{*}{$\begin{array}{l}\text { Radish } \\
\text { cultivar }\end{array}$} & Control & $1 \mathrm{RM}$ & $5 \mathrm{RM}$ & $10 \mathrm{RM}$ & 1RCG & 5RCG & 10RCG & $1 \mathrm{SM}$ & $5 S M$ & $10 \mathrm{SM}$ & 1SCG & 5SCG & 10SCG \\
\hline & \multicolumn{13}{|c|}{ Fresh mass (g) } \\
\hline 'Rowa' & $\begin{array}{c}0.1633 \\
b c \\
\pm 0.03\end{array}$ & $\begin{array}{c}0.1611 \\
b c \\
\pm 0.02\end{array}$ & $\begin{array}{c}0.1837 \\
a b \\
\pm 0.04\end{array}$ & $\begin{array}{c}0.1960 \\
\mathrm{ab} \\
\pm 0.05\end{array}$ & $\begin{array}{c}0.1685 \\
b \\
\pm 0.01\end{array}$ & $\begin{array}{c}0.1721 \\
\text { abc } \\
\pm 0.02\end{array}$ & $\begin{array}{c}0.1734 \\
\text { abc } \\
\pm 0.04\end{array}$ & $\begin{array}{c}0.1868 \\
\mathrm{ab} \\
\pm 0.06\end{array}$ & $\begin{array}{c}0.1962 \\
\mathrm{ab} \\
\pm 0.02\end{array}$ & $\begin{array}{c}0.2042 \\
\mathrm{a} \\
\pm 0.07\end{array}$ & $\begin{array}{c}0.1411 \\
\text { bc } \\
\pm 0.01\end{array}$ & $\begin{array}{c}0.1240 \\
c \\
\pm 0.01\end{array}$ & $\begin{array}{c}0.1865 \\
\mathrm{ab} \\
\pm 0.04\end{array}$ \\
\hline \multirow[t]{2}{*}{ 'Krakowianka' } & $\begin{array}{c}0.2015 \\
\mathrm{a} \\
\pm 0.01 \\
\end{array}$ & $\begin{array}{c}0.2038 \\
\mathrm{a} \\
\pm 0.01 \\
\end{array}$ & $\begin{array}{c}0.1837 \\
\text { ab } \\
\pm 0.03 \\
\end{array}$ & $\begin{array}{c}0.2238 \\
\mathrm{a} \\
\pm 0.06 \\
\end{array}$ & $\begin{array}{c}0.1842 \\
\text { ab } \\
\pm 0.03 \\
\end{array}$ & $\begin{array}{c}0.1824 \\
\mathrm{ab} \\
\pm 0.04 \\
\end{array}$ & $\begin{array}{c}0.1832 \\
\text { ab } \\
\pm 0.04 \\
\end{array}$ & $\begin{array}{c}0.1587 \\
\text { bc } \\
\pm 0.03 \\
\end{array}$ & $\begin{array}{c}0.2091 \\
\mathrm{a} \\
\pm 0.03 \\
\end{array}$ & $\begin{array}{c}0.2121 \\
\mathrm{a} \\
\pm 0.02 \\
\end{array}$ & $\begin{array}{c}0.1924 \\
\mathrm{ab} \\
\pm 0.03 \\
\end{array}$ & $\begin{array}{c}0.2019 \\
a \\
\pm 0.02 \\
\end{array}$ & $\begin{array}{c}0.2053 \\
\mathrm{a} \\
\pm 0.04 \\
\end{array}$ \\
\hline & \multicolumn{13}{|c|}{ Dry mass $(\mathrm{g})$} \\
\hline 'Rowa' & $\begin{array}{c}0.0068 \\
\text { b } \\
\pm 0.001\end{array}$ & $\begin{array}{c}0.0067 \\
\text { b } \\
\pm 0.002\end{array}$ & $\begin{array}{c}0.0100 \\
a \\
\pm 0.001\end{array}$ & $\begin{array}{c}0.0091 \\
a b \\
\pm 0.002\end{array}$ & $\begin{array}{c}0.0081 \\
a b \\
0.001\end{array}$ & $\begin{array}{c}0.0087 \\
a b \\
\pm 0.001\end{array}$ & $\begin{array}{c}0.0097 \\
a b \\
\pm 0.005\end{array}$ & $\begin{array}{c}0.0081 \\
a b \\
\pm 0.002\end{array}$ & $\begin{array}{c}0.0082 \\
a b \\
\pm 0.001\end{array}$ & $\begin{array}{c}0.0094 \\
a b \\
\pm 0.002\end{array}$ & $\begin{array}{c}0.0065 \\
\mathrm{~b} \\
\pm 0.001\end{array}$ & $\begin{array}{c}0.0066 \\
\mathrm{~b} \\
\pm 0.002\end{array}$ & $\begin{array}{c}0.0083 \\
a b \\
\pm 0.001\end{array}$ \\
\hline 'Krakowianka' & $\begin{array}{c}0.0097 \\
a b \\
\pm 0.002 \\
\end{array}$ & $\begin{array}{c}0.0094 \\
\text { ab } \\
\pm 0.002 \\
\end{array}$ & $\begin{array}{c}0.0089 \\
a b \\
\pm 0.001 \\
\end{array}$ & $\begin{array}{c}0.0106 \\
\mathrm{a} \\
\pm 0.001 \\
\end{array}$ & $\begin{array}{c}0.0093 \\
\mathrm{ab} \\
\pm 0.001 \\
\end{array}$ & $\begin{array}{c}0.0091 \\
\mathrm{ab} \\
\pm 0.001 \\
\end{array}$ & $\begin{array}{c}0.0101 \\
\mathrm{a} \\
\pm 0.002 \\
\end{array}$ & $\begin{array}{c}0.0088 \\
\mathrm{ab} \\
\pm 0.001 \\
\end{array}$ & $\begin{array}{c}0.0095 \\
\mathrm{ab} \\
\pm 0.001 \\
\end{array}$ & $\begin{array}{c}0.0109 \\
\mathrm{a} \\
\pm 0.001 \\
\end{array}$ & $\begin{array}{c}0.0086 \\
\mathrm{ab} \\
\pm 0.002 \\
\end{array}$ & $\begin{array}{c}0.0090 \\
a b \\
\pm 0.001 \\
\end{array}$ & $\begin{array}{c}0.0093 \\
\text { ab } \\
\pm 0.002 \\
\end{array}$ \\
\hline & \multicolumn{13}{|c|}{ Dry mass (\%) } \\
\hline 'Rowa' & $\begin{array}{c}4.15 \\
\text { b } \\
\pm 0.97\end{array}$ & $\begin{array}{c}4.17 \\
\text { b } \\
\pm 0.96\end{array}$ & $\begin{array}{c}5.42 \\
a \\
\pm 0.93\end{array}$ & $\begin{array}{c}4.65 \\
\mathrm{ab} \\
\pm 0.54\end{array}$ & $\begin{array}{c}4.78 \\
\mathrm{ab} \\
\pm 0.30\end{array}$ & $\begin{array}{c}5.03 \\
a \\
\pm 0.61\end{array}$ & $\begin{array}{c}5.62 \\
a \\
\pm 1.50\end{array}$ & $\begin{array}{c}4.33 \\
\text { b } \\
\pm 0.63\end{array}$ & $\begin{array}{c}4.17 \\
\text { b } \\
\pm 1.23\end{array}$ & $\begin{array}{c}4.60 \\
\mathrm{ab} \\
\pm 0.35\end{array}$ & $\begin{array}{c}4.61 \\
\mathrm{ab} \\
\pm 1.23\end{array}$ & $\begin{array}{c}5.29 \\
a \\
\pm 0.49\end{array}$ & $\begin{array}{c}4.43 \\
\text { b } \\
\pm 0.55\end{array}$ \\
\hline 'Krakowianka' & $\begin{array}{c}4.80 \\
\text { ab } \\
\pm 0.74\end{array}$ & $\begin{array}{c}4.60 \\
\mathrm{ab} \\
\pm 0.86\end{array}$ & $\begin{array}{c}4.84 \\
\mathrm{ab} \\
\pm 1.91\end{array}$ & $\begin{array}{c}4.71 \\
\text { ab } \\
\pm 1.57\end{array}$ & $\begin{array}{c}5.06 \\
a \\
\pm 0.37\end{array}$ & $\begin{array}{c}5.01 \\
a \\
\pm 0.77\end{array}$ & $\begin{array}{c}5.52 \\
\mathrm{a} \\
\pm 1.59\end{array}$ & $\begin{array}{c}5.54 \\
\mathrm{a} \\
\pm 0.22\end{array}$ & $\begin{array}{c}4.53 \\
\text { ab } \\
\pm 0.39\end{array}$ & $\begin{array}{c}5.15 \\
a \\
\pm 0.29\end{array}$ & $\begin{array}{c}4.47 \\
\text { ab } \\
\pm 0.78\end{array}$ & $\begin{array}{c}4.44 \\
\text { ab } \\
\pm 0.78\end{array}$ & $\begin{array}{c}4.55 \\
\text { ab } \\
\pm 0.53\end{array}$ \\
\hline & \multicolumn{13}{|c|}{ Water content $(\%)$} \\
\hline 'Rowa' & $\begin{array}{c}95.85 \\
\mathrm{a} \\
\pm 0.97\end{array}$ & $\begin{array}{c}95.83 \\
\mathrm{a} \\
\pm 0.96\end{array}$ & $\begin{array}{c}94.58 \\
\text { b } \\
\pm 0.93\end{array}$ & $\begin{array}{c}95.35 \\
\mathrm{a} \\
\pm 0.54\end{array}$ & $\begin{array}{c}95.22 \\
\mathrm{a} \\
\pm 0.30\end{array}$ & $\begin{array}{c}94.98 \\
\text { ab } \\
\pm 0.61\end{array}$ & $\begin{array}{c}94.38 \\
\text { ab } \\
\pm 1.50\end{array}$ & $\begin{array}{c}95.67 \\
\mathrm{a} \\
\pm 0.63\end{array}$ & $\begin{array}{c}95.83 \\
\text { a } \\
\pm 0.44\end{array}$ & $\begin{array}{c}95.40 \\
\mathrm{a} \\
\pm 1.23\end{array}$ & $\begin{array}{c}95.39 \\
\mathrm{a} \\
\pm 0.35\end{array}$ & $\begin{array}{c}94.71 \\
\text { ab } \\
\pm 1.23\end{array}$ & $\begin{array}{c}95.47 \\
\text { a } \\
\pm 0.49\end{array}$ \\
\hline 'Krakowianka' & $\begin{array}{c}95.20 \\
\text { a } \\
\pm 0.55\end{array}$ & $\begin{array}{c}95.40 \\
\text { a } \\
\pm 0,74\end{array}$ & $\begin{array}{c}95.16 \\
\mathrm{a} \\
\pm 0.86\end{array}$ & $\begin{array}{c}95.29 \\
\mathrm{a} \\
\pm 1.95\end{array}$ & $\begin{array}{c}94.94 \\
\text { ab } \\
\pm 0.16\end{array}$ & $\begin{array}{c}94.99 \\
\text { ab } \\
\pm 0.37\end{array}$ & $\begin{array}{c}94.48 \\
\text { ab } \\
\pm 0.77\end{array}$ & $\begin{array}{c}94.46 \\
\mathrm{ab} \\
\pm 1.59\end{array}$ & $\begin{array}{c}95.47 \\
\text { a } \\
\pm 0.22\end{array}$ & $\begin{array}{c}94.85 \\
\mathrm{ab} \\
\pm 0.39\end{array}$ & $\begin{array}{c}95.53 \\
\text { a } \\
\pm 0.29\end{array}$ & $\begin{array}{c}95.56 \\
\mathrm{a} \\
\pm 0.78\end{array}$ & $\begin{array}{c}95.45 \\
a \\
\pm 0.53\end{array}$ \\
\hline
\end{tabular}

Control - distilled water, 1, 5, 10RM - 1\%, 5\%, 10\% extract from root plant grown on meadow, 1, 5, 10RCG - 1\%, $5 \%, 10 \%$ extract from root plant grown on calcareous grasslands, 1, 5, 10SM - 1\%, 5\%, 10\% extract from shoot plant grown on meadow, 1, 5, 10SCG - 1\%, 5\%, 10\% extract from shoot plant grown on calcareous grasslands; mean values $(\mathrm{n}=5, \pm \mathrm{SE})$ marked different letter differ statistically (in row) according to Duncan test at $\mathrm{p}<0.05$

\section{Fresh, dry mass, and water content}

The fresh mass of 'Rowa' radish seedlings was significantly highest for the seeds watered with $10 \%$ of the shoots from the meadows, compared to the control. Significantly the lowest fresh mass value of seedlings was found for seeds watered with $5 \%$ of $F$. vulgaris shoot extracts from calcareous grasslands. For the 'Krakowianka', no statistically significant differences in the fresh mass values were observed between the control and the extracts from the dropwort organs used (Table 2).

In the case of dry mass, its significant increase was demonstrated for seedlings of 'Rowa' cultivar grown from seeds watered with $5 \%$ root extract from meadow areas, compared to the control. In other cases, no significant differences in the values of this parameter were observed.

In general, for the 'Rowa' cultivar a percentage of the dry mass index was significantly higher for seedlings grown on root extracts than shoot extracts. For the 'Krakowianka', no differences in the values of this index were found. The total water content was the lowest in 'Rowa' seedlings grown from seeds watered with $5 \%$ root extracts from the meadow, compared to the control and all tested extracts. For the 'Krakowianka' cultivar, no changes in the percentage of water content were observed (Table 2). 


\section{Electrolyte leakage}

The percentage of electrolyte leakage was the highest in the control for both radish cultivars. For radish seedlings watered with $1 \%$ root extracts from meadows, similar values of this parameter were observed. The lowest degree of cell membrane destabilisation, for seedlings watered with $10 \%$ of the root extracts from meadows and $5 \%$ of the shoot extracts from calcareous grasslands, was measured. In the remaining extracts, the electrolyte leakage values were significantly lower than in the control group (Figure 6).

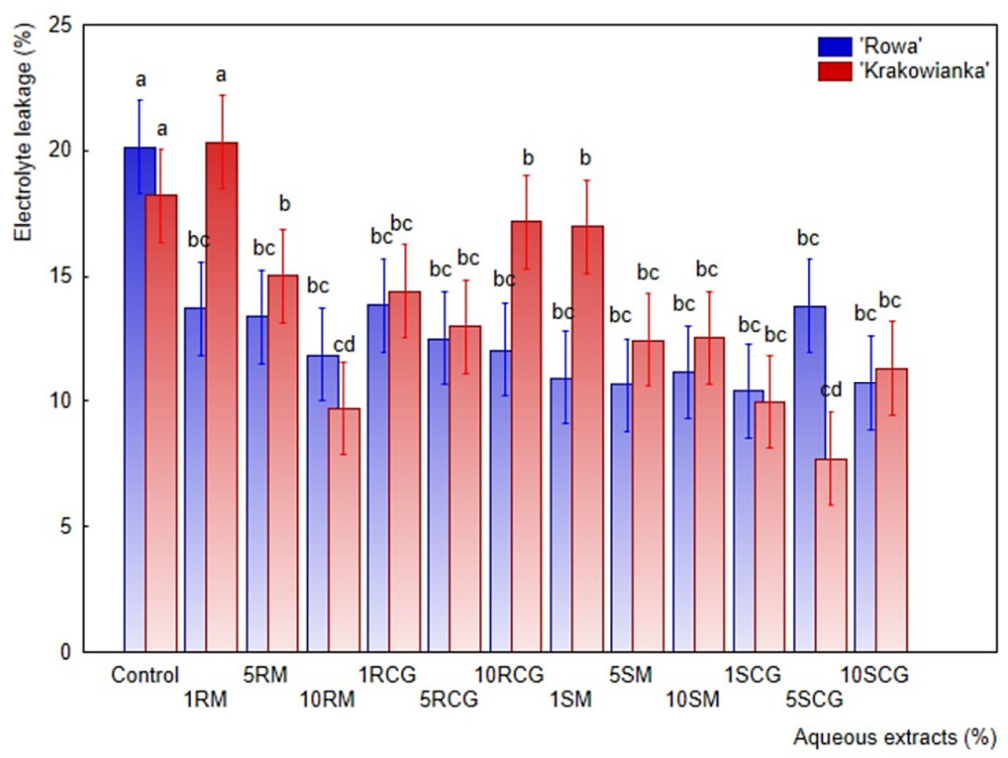

Figure 6. Electrolyte leakage of Raphanus sativus L. var. radicula Pers. 'Rowa' and 'Krakowianka' seedlings after 7 days of watering with aqueous extracts from roots and shoots Filipendula vulgaris Moench with different concentrations $(1,5,10 \%)$ and under control conditions

RM - extract from root plant grown on the meadow, SCG - extract from shoot plant grown on calcareous grasslands; mean values $(\mathrm{n}=3, \pm \mathrm{SE})$ marked different letter differ statistically according to Duncan test at $\mathrm{p}<0.05$

\section{Discussion}

A growing interest in the use of allelopathic plants is observed, both in the direct use as plants controlling weeds as well as attempts to isolate specific compounds of high biological activity as natural herbicides (Duke et al., 2002; Singh et al., 2003; Golisz et al., 2004). The medicinal plants are considered an important source of secondary metabolites and recently they are studied in case of herbicidal potential in controlling major crop weeds for sustainable agricultural production. The possibility to have more bioactive compounds in medicinal plants than in other plants stand behind the special interest in this group of species. As a result of the researches, a considerable number of examined medicinal plants exhibited allelopathic properties (Islam et al., 2018). The studies investigated the suppression of extracts from medicinal plants on model plants like lettuce (e.g. Amini et al., 2016), radish (e.g. Haugland and Brandsaeter, 1996), chosen weeds (e.g. Boonmee and Kato-Noguchi, 2019), or other cultivated species (e.g. Puła et al., 2020). This study showed the allelopathic effect of aqueous extracts from Filipendula vulgaris roots and shoots on germination and early stages of growth of model radish seeds in two cultivars. The percentage of seed germination was decreased with the increase in the concentration of aqueous extracts. The smallest differences were found between the control and $1 \%$ of Filipendula vulgaris root and shoot extracts regardless of the habitats. The highest inhibition of germination capacity seeds was observed on the $10 \%$ dropwort extracts. On the root extracts, more percentage of germinated seeds were observed than shoots extracts (Figure 2). In general, the performed determinations of germination indexes 
showed that extracts with $1 \%$ concentration significantly stimulated seed germination. On the other hand, dropwort extracts with a concentration of $10 \%$ had an inhibitory effect on the germination capacity, regardless of the radish cultivars (Table 1). Germination depends on many factors biochemical and molecular, from the activation of the metabolism of the embryo embedded in the seed, the quality of the RNA and the integrity of the DNA, plant hormones (Koorneef et al., 2002; Rajjou et al., 2012). In the germination process many enzymes participate, which activity in the presence of allelopathic compounds can be destabilized. Reducing the activity of hydrolytic enzymes means that the seeds are not able to provide enough energy for germinating seeds, which reduces the germination capacity and causes the formation of weakened seedlings (Zandi et al., 2018; Możdżeń et al., 2020). Allelochemical substances increase the vacuolization of cells, which is accompanied by a reduction in the number of mitochondria, the density of ribosomes, and dictiosomes (Burgos et al., 2004). The seed coat can also play an important role, as it protects the seed against harmful external factors and mechanical damage (Rzepka and Możdżeń, 2016; Stachurska-Swakoń et al., 2018; Mazur, 2019). It is easily permeable to oxygen and water. The lower concentration of allelopathic compounds in $1 \%$ extracts means that more water is absorbed by the seeds, hence perhaps a positive influence of extracts of low concentrations on the values of selected germination indexes, and no differences between the control samples and dropwort extracts was observed. Root or leaf extracts may also contain diverse phytotoxins with different modes of action (Inderjit et al., 2007). Therefore, it can be assumed that the different reactions of radish seeds result from a different concentration of allelochemical compounds in aqueous extracts from roots and shoots.

Understanding the mechanisms of action of allelochemical compounds on plants is difficult, if only because they belong to different chemical classes and it is difficult to expect them to act in the same way. It is assumed that allelochemical compounds affect comprehensively. Moreover, allelochemicals present in the plant after reaching the rhizosphere can be quickly converted into compounds with greater or lesser activity. Therefore, it is important to assess the concentration and form in which the allelochemical is available for the species. It should also be considered how the habitat, physical, chemical, and biological soil factors, climatic conditions influence the qualitative effects and quantitative concentration of a given compound (Kaur et al., 2005). There is a close correlation between the occurrence of environmental stresses that directly shape plant growth and stimulation of phytotoxic production (Saxena et al., 1996; San Emeterio et al., 2007; Gioria and Osborne, 2014). As a result, it leads to morphogenetic changes and affects the size and quality of the crop (Rice, 1984; Einheling, 1995). The growth of plants is a very sensitive process to the presence of allelochemical compounds. Allelochemical compounds reveal their effect already during seed swelling, affecting the cells of the seed coat and aleurone layer, mitotic cell division, shaping cell organelles, and also elongation. They usually distort the cell walls in the cells of the apex of the root growth and contribute to the accumulation of lipids in the cytoplasm of the cells (Wójcik-Wojtkowiak et al., 1998; Kaur et al., 2005). According to Burgos et al. (2004), inhibition of seed cell growth is caused by disturbances in lipid metabolism and the reduction of protein synthesis. In our study, when considering biometry, a stimulating effect of $1 \%$ of $F$. vulgaris root and shoot extracts obtained from the meadow and calcareous grassland on hypocotyle length was observed. As the concentrations increased, the elongation of seedling organs was inhibited (especially on 10\% extracts) (Figures 3-5).

The aqueous extracts of the dropwort roots and shoots did not significantly influence the fresh and dry mass (Table 2). The allelopathic effect of $F$. vulgaris depended on the concentration of the extracts. Only the 'Rowa' presented a slight increase in the fresh mass of seedlings on $5 \%$ of $F$. vulgaris shoot extracts from calcareous grasslands and dry mass on 5\% root extract from meadow areas. Similarly, no differences in water content in seedlings were found. These results suggest the presence of different concentrations of soluble allelochemical substances in the aqueous extracts of dropwort. The higher toxicity of meadow extracts than calcareous grassland is probably related to the presence of more amounts of allelopathic compounds in plants. Their toxicity may be due to also a synergistic action, and not only to the action of individual substances. The concentration of a chemical in solution may be significantly less than the concentration of a single allelopathic substance required to produce a plant growth inhibitory effect (Xuan et al., 2004; Inderjit et al., 2007). 
Allelopathic compounds have a primary effect on cytoplasmic membranes. Their damage affects the entire metabolism and all physiological processes (Wójcik-Wojtkowiak et al., 1998). In this research, a significant reduction in the degree of destabilization of cell membranes, regardless of the extract used, was found. However, it was lower than in the control, which is an interesting result (Figure 6). This radish seedling response may be due to the lower content of phenolic compounds in leaves than in flowers (Bączek et al., 2012). Recent studies on the extracts of $F$. vulgaris revealed that they contain several classes of phenolic compounds (Katanić et al., 2018). Flavonoid glycosides have been described as a major constituent of the upper parts of dropwort, and catechin and procyanids were identified as a major constituent of roots (Oszmiański et al., 2007). Such a reaction of seedlings also depends on the harvest period of the plants from which the extracts were prepared. Bączek et al. (2012) have found seasonal variation in the content of phenolic compounds in the upper parts of the species, they were higher at the beginning of flowering as compared with the full flowering stage. Additionally, the content of catechins and polyphenolic acids found in the roots did not change during the season. In the present study, $F$. vulgaris plants were harvested in the late generative stage, which probably reduced the electrolyte leakage. On the other hand, we cannot exclude the probable presence of calcium in $F$. vulgaris extracts, the role of which in membrane stabilization is known (Hepler, 2005).

It is commonly believed that plants have a negative allelopathic effect on other plants, but some of them also play a positive role. Some plants contribute to the protection of soils against erosion, improve their fertility, and have a positive effect on the condition of the soil microflora. Allelochemical compounds secreted by plants that stimulate the growth of other plants are also known. However, they constitute a small group compared to allelochemicals compounds inhibitory (Haupt et al., 2001; Goufo et al., 2014; Kopczewski, 2014). Our study pointed at the inhibiting effect of all extracts on germination capacity. At low concentrations of aqueous extracts from the roots and shoots of dropwort the germination indexes had higher values than control. The biometric and biomass parameters of seedlings were generally stimulated by these extracts, regardless of the plant harvesting habitats, organs, and concentrations, as compared to the control seedlings. Therefore, it is necessary to conduct further research on the allelopathic interactions of plants to understand their effects not only on seed germination but also their physiology and plants grown from them. Additionally, it seems necessary to study the chemical composition to identify chemical compounds responsible for this type of reaction and to strive for their practical application in various fields. Multidisciplinary studies involving plant ecology, genetics, physiology, biochemistry, soil science, and microbiology can answer the key questions of if and how allelopathy influences plant interactions.

\section{Conclusions}

Plants, in order to survive in the natural environment, developed many defence mechanisms, including the ability to synthesize and secrete compounds with allelopathic potential. In the literature, there are various groups of allelochemical compounds as well as the sources of their release into the environment. The release of substances with the allelopathic potential to the environment may inhibit or stimulate seed germination and plant growth. Their impact on other organisms depends on their chemical nature, the species they affect, and the prevailing environmental conditions.

This study revealed the inhibiting effect of aqueous extracts from Filipendula vulgaris organs on the germination of two cultivars of radish seeds. However, the stimulating effect of $1 \%$ of aqueous extracts from dropwort roots and shoots on the germination indexes was revealed. As the concentration of allelopathic compounds in the extracts increased, a decrease in the values of germination indexes was observed, compared to the control. Similarly, the biometry of radish seedlings revealed a positive effect of extracts with low concentrations, as opposed to extracts with a higher content of allelopathic substances (especially, 10\% extracts). Observations of changes in the length of the root organs, hypocotyls, and whole seedlings were similar, regardless of the type and concentration of the extract, concerning seedlings grown on distilled water. 
For the 'Rowa' cultivar, an increase in the fresh mass of seedlings with an increase in the concentration of extracts was demonstrated, in contrast to the 'Krakowianka'. The dry mass values generally no significant differences were changed. The exception was 'Rowa' cultivar grown from seeds watered with 5\% root extract from meadow areas, where the dry mass was lower. The percentage of dry mass depended on the concentration and type of extract used for watering the radish seeds. A positive effect of $5 \%$ and $10 \%$ extracts was observed, regardless of the organ used in preparing the extract. A positive effect of all dropwort extracts on the electrolyte leakage was demonstrated.

\section{Authors' Contributions}

ASS planned and designed the research; MF, KM performed the experiment; ASS, KM, BBK, MF analysed the data and prepared figures and tables, ASS, KM, BBK wrote the manuscript. All authors read and approved the final manuscript.

\section{Acknowledgements}

This work was supported by the Institute of Botany at the Jagiellonian University, Kraków, Poland (N18/MNS/000009).

\section{Conflict of Interests}

The authors declare that there are no conflicts of interest related to this article.

\section{References}

Amini S, Azizi M, Joharchi MR, Moradinezhad F (2016). Evaluation of allelopathic activity of 68 medicinal and wild plant species of Iran by Sandwich method. International Journal of Horticultural Science and Technology 3:243-253. https://doi.org/10.22059/ijhst.2016.62923

Atanasov AG, Waltenberger B, Pferschy-Wenzig EM, Linder T, Wawrosch C, Uhrin P, ... Stuppner H (2015). Discovery and resupply of pharmacologically active plant-derived natural products: A review. Biotechnology Advances 33(8):1582-1614. https://doi.org/10.1016/j.biotechadv.2015.08.001

Bączek K, Cygan M, Przybył JL, Kosakowska O, Węglarz Z (2012). Seasonal variation of phenolics content in above-and underground organs of dropwort (Filipendula vulgaris Moench). Herba Polonica 58(3):24-32.

Bączek K, Przybył JL, Angielczyk M, Kuczerenko A, Pelc M, Podyma W, Węglarz Z (2011). Accumulation of phenolic compounds in leaves and underground organs of dropwort (Filipendula vulgaris L.). Acta Horticulture 925:147150. https://doi.org/10.17660/ActaHortic.2011.925.20

Batish DR, Singh HP, Kohli RK, Dawra GP (2006). Potential of allelopathy and allelochemicals for weed management. Food Prod Press, New York.

Birkett MA, Chamberlain K, Hoopera M, Pickett JA (2001). Does allelopathy offer real promise for practical weed management and for explaining rhizosphere interactions involving higher plants? Plant Soil 232:31-39. https://doi.org/10.1023/A:1010325801256

Boonmee S, Kato-Noguchi H (2019). Comparative study on allelopathic potential of medicinal plants against the growth of cress and barnyard grass. Environmental Control in Biology 57:75-81. https://doi.org/10.2525/ECB.57.75

Burgos NR, Talbert RE, Kimk S, Kuky I (2004). Growth inhibition and root ultrastructure of cucumber seedlings exposed to allelochemicals from rye (Secale cereale). Journal of Chemical Ecology 30:671-689. https://doi.org/10.1023/B:JOEC.0000018637.94002.ba 
Capecka E, Geszprych A, Przybył JL, Kunicki E, Binder A, Bączek K, Węglarz Z (2012). Accumulation of phenolic compounds in underground organs of dropwort (Filipendula vulgaris Moench). Acta Scientiarum Polonorum, Hortorum Cultus 11(4):101-109.

Clapham AR, Tutin TG, Moore DM (1987). Flora of the British Isles. Cambridge University Press, Cambridge.

Daizy R, Batish DR, Singh HP, Kohli RK, Saxena DB, Dakora FD (1995). Plant flavonoids: biological molecules for useful exploitation. Australian Journal of Plant Physiology 22(87):87-99. https://doi.org/10.1071/PP9950087

Dayan FE, Cantrell CL, Duke SO (2009). Natural products in crop protection. Bioorganic and Medicinal Chemistry 17:4022-4034.

Dubiel E, Stachurska A, Gawroński S (1999). Nieleśne zbiorowiska roślinne Magurskiego Parku Narodowego (Beskid Niski) (Non-forest communities of the Magura National Park (Beskid Niski Mts.)). Zeszyty Naukowe Uniwersytetu Jagiellońskiego, Prace Botaniczne 33:1-60.

Duke SO, Dayan FE, Rimando AM, Schrader KK, Aliotta G, Oliva A (2002). Chemicals from nature for weed management. Weed Sciences 50:138-151. https://www.jstor.org/stable/4046356

Einhelling, F.A. (1995). Mechanism of action of allelochemicals in allelopathy. In: Inderjit KMM, Dakshini FA (Eds.), Allelopathy, Organisms, Processes and Applications; ASC Symp., Ser. 582, Washington, pp 96-116.

Fujii Y, Parvez SS, Parvez MM, Ohmae Y, Uda O (2003). Screening of 239 medicinal plant species for allelopathic activity using the sandwich method. Weed Biology Management 3:233-241. https://doi.org/10.1046/j.14446162.2003.00111.x

Gioria M, Osborne BA (2014). Resource competition in plant invasions: emerging patterns and research needs. Frontiers in Plant Science 5:501. https://doi.org/10.3389/fpls.2014.00501

Golisz A, Gawroński SW, Gawrońska H (2004). Allelopathic activity of buckwheat on quackgrass growth and development. Zeszyty Problemowe Postępów Nauk Rolniczych 496:315-324.

Goufo P, Pereira J, Moutinho-Pereira J, Correia C, Figueiredo N, Carranca C, Rosa E, Trindade H (2014). Rice (Oryza sativa L.) phenolic compounds under elevated carbon dioxide $\left(\mathrm{CO}_{2}\right)$ concentration. Environmental and Experimental Botany 99:28-37. https://doi.org/10.1016/j.envexpbot.2013.10.02

Haugland E, Brandsaeter LO (1996). Experiments on bioassay sensitivity in the study of allelopathy. Journal of Chemical Ecology 22:1845-1859. https://doi.org/10.1007/BF02028508

Haupt S, Oparka KJ, Sauer N, Neumann S (2001). Macromolecular trafficking between Nicotina tabacum and the holoparasite Cuscuta reflexa. Journal of Experimental Botany 52:173-177. https://doi.org/10.1093/jexbot/52.354.173

Hepler PK (2005). Calcium: a central regulator of plant growth and development. The Plant Cell 17(8):2142-2155. https://doi.org/10.1105/tpc.105.032508

Imbrea IM, Butnariu M, Nicolin AL, Imbrea F (2010). Determining antioxidant capacity of extracts of Filipendula vulgaris Moench from south-western Romania. Journal of Food, Agriculture and Environment 8:111-116.

Inderjit, Weston LA, Duke SO (2007). Challenges, achievements and opportunities in allelopathy research. Journal of Plant Interactions 1(2):69-81. https://doi.org/10.1080/17429140600622535

Islam AKM, Yeasmin S, Qasem JRS, Juraimi AS, Anwar MP (2018). Allelopathy of medicinal plants: current status and future prospects in weed management. Agricultural Sciences 9:1569-1588. https://doi.org/10.4236/as.2018.912110

Kamboj VP (2000). Herbal Medicine. Current Science 78:35-39.

Katanić J, Mihailović V, Stanković N, Boroja T, Mladenović M, Solujić S, ... Vrvić MM (2015). Dropwort (Filipendula hexapetala Gilib.): potential role as antioxidant and antimicrobial agent. Experimental and Clinical Sciences Journal 14:1-20. https://dx.doi.org/10.17179\%2Fexcli2014-479

Katanić J, Pferschy-Wenzing EM, Mihailović V, Boroja T, Pan S-P, ... Bauer R (2018). Phytochemical analysis and antiinflammatory effects of Filipendula vulgaris Moench extracts. Food and Chemical Toxicology 122:151-162. https://doi.org/10.1016/j.fct.2018.10.001

Kaur H, Inderjit, Kaushik S (2005). Cellular evidence of allelopathic interference of benzoic acid to mustard (Brassica juncea L.) seedling growth. Plant Physiology and Biochemistry 43:77-81. https://doi.org/10.1016/j.plaphy.2004.12.007

Klimešová J, Klimeš L, (2006). Clo-Pla 3 Database of clonal growth of plants from Central Europe, available from: http://clopla.butbn.cas.cz/

Koorneef M, Bentsink L. Hilhorst H (2002). Seed dormancy and germination. Current Opinion in Plant Biology 5(1):3336. https://doi.org/10.1016/S1369-5266(01)00219-9 
Kopczewski T (2014). Allelopatia - czy rośliny potrafią się komunikować? (Allelopathy - can plants communicate?) Edukacja biologiczna i środowiskowa 3:3-8. (In Polish)

Kostrakiewicz-Gierałt K, Stachurska-Swakoń A (2017). The influence of habitat conditions on the abundance and selected traits of the rare medicinal plant species Filipendula vulgaris Moench. Ecological Questions 25:9-18. http://dx.doi.org/10.12775/EQ.2017.001

Kupczak K (2011). Odmiany warzyw. Uprawy polowe 2011-2012. PlantPress, Kraków.

Maksimović Z, Petrović S, Pavlović M, Kovačević N, Kukić J (2007). Antioxidant activity of Filipendula hexapetala flowers. Fitoterapia 78:265-267.

Mazur A (2020). The role of seed coat in the germination and early stages of growth of bean (Phaseolus vulgaris L.) in the presence of chickweed (Stellaria media (L.) Vill.). Annales Universitatis Paedagogicae Cracoviensis Studia Naturae 4:103-118. https://doi.org/10.24917/25438832.4.6

McErlich A, Boydston RA (2013). Current state of weed management in organic and conventional cropping systems. In: Young SL, Pierce FJ, editors. Automation, the future of weed control in cropping systems. Springer Publishing, New York pp 11-32.

Medwecka-Kornaś A, Kornaś J, Pawłowski B (1966). Survey of the most important plant associations in Poland. In: Szafer W (Ed.), The Vegetation of Poland, Pergamon Press, pp 294-509.

Movsumov IS, Garaev EE, Herbette G, Baghdikian B, Ollivier E, Elias R, (...), Garaev EA (2017). Flavanoids of Acasia dealbata and Filipendula vulgaris growing in Azerbaijan. Chemistry of Natural Compounds 53(4):754-755. https://doi.org/10.1007/s10600-017-2111-3

Mowszowicz J (1985). Przewodnik do oznaczania krajowych roślin zielarskich. PWRiL, Warszawa.

Możdżeń K, Barabasz-Krasny B, Puła J, Lepiarczyk A. Zandi P (2018). Wpływ stresu zasolenia i suszy na wczesny rozwój wybranych gatunków pastewnych (The influence of salinity and drought stress on the early growth of selected fodder plant species). Fragmenta Agronomica 35(3):77-88. https://doi.org/10.26374/fa.2018.35.3

Możdżeń K, Barabasz-Krasny B, Stachurska-Swakoń A, Zandi P, Puła J, Wang Y, Turisova I (2020). Allelopathic interaction between two common meadow plants: Dactylis glomerata L. and Trifolium pratense L. Biologia 75:653-663. https://doi.org/10.2478/s11756-020-00438-6

Możdżeń K, Rzepka A (2016). Rola łupiny nasiennej podczas kiełkowania i wzrostu nasion bobu (Vicia faba L.) w obecności siarczanu ołowiu (The role of the seed coat on germination and growth of broad beans (Vicia faba L.) in lead sulfate conditions). Agronomy Science 71:55-65. (In Polish)

Olennikov DN, Kruglova MY (2013). A new quercetin glycoside and other phenolic compounds from the genus Filipendula. Chemistry of Natural Compounds 49:610-616. https://doi.org/10.1007/s10600-013-0691-0

Olennikov DN, Kruglova MY (2013). A new quercetin glycoside and other phenolic compounds from the genus Filipendula. Chemistry of Natural Compounds 49:610-616. https://doi.org/10.1007/s10600-013-0691-0

Oszmianski J, Wojdylo A, Lamer-Zarawska E, Swiader K (2007). Antioxidant tannins from Rosaceae plant roots. Food Chemistry 100:579-583. https://doi.org/10.1016/j.foodchem.2005.09.086

Pavlovic M, Petrovic S, Ristic M, Maksimovic Z, Kovacevic N (2007). Essential oil of Filipendula hexapetala. Chemistry of Natural Compounds 43(2):228-229. https://doi.org/10.1007/s10600-007-0088-Z

Puła J, Zandi P, Stachurska-Swakoń A, Barabasz-Krasny B, Możdżeń K, Wang Y (2020). Influence of alcoholic extracts from Helianthus annnus L. roots on the photosynthetic activity of Sinapis alba L. cv. Barka plants. Acta Agriculturae Scandinavica, Section B, 70(1):8-13. https://doi.org/10.1080/09064710.2019.1661509

Rajjou L, Duval M, Gallardo K, Catusse J, Bally J, Job C, Job D (2012). Seed germination and vigor. Annual Review of Plant Biology 63:507-533. https://doi.org/10.1146/annurev-arplant-042811-105550

Rice EL (1984). Allelopathy, 2nd ed.; Academic Press: Orlando, Florida.

Samardžić S, Tomić M, Pecikoza U, Stepanović-Petrović R, Maksimović Z (2016). Antihyperalgesic acitvity of Filipendula ulmaria (L.) Maxim. and Filipendula vulgaris Moench in rat model of inflammation. Journal of Ethnopharmacology 193:652-656. https://doi.org/10.1016/j.jep.2016.10.024

San Emeterio L, Damgaard C, Canals RM (2007). Modelling the combined effect of chemical interference and resource competition on the individual growth of two herbaceous populations. Plant and Soil 292:95-103. https://doi.org/10.1007/s11104-007-9205-9

Saxena A, Singh DV, Joshi NL (1996). Autotoxic effects of pearl millet aqueous extracts on seed germination and seedling growth. Journal of Arid Environment 33:255-260. https://doi.org/10.1006/jare.1996.0061 
Shilova IV, Korotkova EI (2017). Biologically active substances from dropwort (Filipendula vulgaris) and assessment of their antioxidant properties. Pharmaceutical Chemistry Journal 51(7):602-605. https://doi.org/10.1007/s11094-017-1660-X

Shilova IV, Suslov NI (2015). Nootropic effect of Meadowsweet (Filipendula vulgaris) extracts. Bulletin of Experimental Biology and Medicine 158(5):659-663. https://doi.org/10.1007/s10517-015-2841-9

Shilova IV, Suslov NI, Amelchenko VP (2015). Nootropic effects of Filipendula vulgaris Moench water extract fractions. Bulletin of Experimental Biology and Medicine 159(3):376-379. https://doi.org/10.1007/s10517-015-2967-9

Singh HP, Batish DR, Kohli RK (2003). Allelopathic interactions and allelochemicals: New possibilities for sustainable weed management. Critical Review in Plant Sciences 22(3-4):239-311.

Skiba S, Drewnik M, Szymański W, Żyła M (2008). Mapa gleb Krakowa. Skala 1:20 000. [Soil map of Krakow]. Instytut Geografii i Gospodarki Przestrzennej Uniwersytetu Jagiellońskiego.

Skrzypek E, Repka P, Stachurska-Swakoń A, Barabasz-Krasny B, Możdżeń K (2016). Seedlings growth of common sunflower under influence of peppermint extract. Modern Phytomorphology 9:69-73.

Stachurska-Swakoń A, Barabasz-Krasny B, Klasa A, Palaczyk A (2018). Reduced plant fitness by pre-dispersal seed predation in the threatened plant species Cirsium decussatum. Seed Science Research 28(2):123-130. https://doi.org/10.1017/S0960258518000107

Tal A. (2018). Making conventional agriculture environmentally friendly: moving beyond the glorification of organic agriculture and the demonization of conventional agriculture. Sustainability 10:1078. https://doi.org/10.3390/su10041078

Tapsell LC, Hemphill I, Cobiac L, Patch CS, Sullivan DR, Fenech M, ... Inge KE (2006). Health benefits of herbs and spices: the past, the present, the future. The Medical Journal of Australian 185(4):4-24. https://doi.org/10.5694/j.1326-5377.2006.tb00548.x

Towpasz K, Stachurska-Swakon A (2009). Occurrence of Sesleria uliginosa (Poaceae) in the communities of the Caricetalia davallianae order in the Nida Basin territory (Małopolska Upland). Fragmenta Floristica et Geobotanica Polonica 16(2):305-316.

Towpasz K, Stachurska-Swakoń A (2011). Occurrence of Sesleria uliginosa (Poaceae) in the xerothermic grasslands (Festuco-Brometea) in the Nida Basin territory (Małopolska Upland)]. Fragmenta Floristica et Geobotanica Polonica 18(2):321-330.

Towpasz K, Stachurska-Swakoń A (2012). Seslerio uliginosae-Scorzoneretum purpureae (Festuco-Brometea class) in the Nida Basin (Małopolska Upland) after 90 years. Acta Societatis Botanicorum Poloniae 81(3):167-173. https://doi.org/10.5586/asbp.2012.022

Tucakov J (1973). Lečenje biljem: fitoterapija. Beograd: Izdavačko preduzeće Rad.

Vengerovsky AI, Suslov NI, Kaygorodsev AV (2011). Effect of Meadowsweet (Filipendula vulgaris) extract on bioenergetics of the brain during experimental post-hypoxic encephalopathy. Bulletin of Experimental Biology and Medicine 151:421-424. https://doi.org/10.1007/s10517-011-1346-4

Verma S, Singh SP (2008). Current and future status of herbal medicines. Veterinary World 1:347-350. https://doi.org/10.5455/vetworld.2008.347-350

Wakdikar S (2004). Global Health Care Challenge: Indian Experiences and New Prescriptions. Electronic Journal of Biotechnology 7:2-3. https://doi.org/10.2225/vol7-issue3-fulltext-5

Weidema IR, Magnussen LS, Philipp M (2000). Gene flow and mode of pollination in a dry grassland species, Filipendula vulgaris (Rosaceae). Heredity 84(3):311-320. https://doi.org/10.1046/j.1365-2540.2000.00669.x

Wójcik-Wojtkowiak D, Politycka B, Weyman-Kaczmarkowa W (1998). Allelopatia. Poznań: Wydawnictwo Akademii Rolniczej w Poznaniu.

Zając M, Zając A (2009). Elementy geograficzne rodzimej flory Polski (The geographical elements of native flora of Poland). Institute of Botany, Jagiellonian University, Kraków, Poland.

Zandi P, Barabasz-Krasny B, Stachurska-Swakoń A, Puła J, Możdżeń K (2018). Allelopathic effects of Stellaria media(L.) Vill. on germination and early stages of growth of Raphanus sativus var. radicula. Annales Universitatis Paedagogicae Cracoviensis Studia Naturae 3:90-99. https://doi.org/10.24917/25438832.3.7

Xuan TD, Tsuzuki E, Tawata S, Khanh TD (2004). Methods to determine allelopathic potential of crop plants for weed control. Allelopathy Journal 13:149-164. 
Fecowicz M et al. (2020). Not Bot Horti Agrobo 48(4):2032-2049

OPEN ACCESS

(c) (2)

The journal offers free, immediate, and unrestricted access to peer-reviewed research and scholarly work. Users are allowed to read, download, copy, distribute, print, search, or link to the full texts of the articles, or use them for any other lawful purpose, without asking prior permission from the publisher or the author.

License - Articles published in Notulae Botanicae Horti Agrobotanici Cluj-Napoca are Open-Access, distributed under the terms and conditions of the Creative Commons Attribution (CC BY 4.0) License. (C) Articles by the authors; UASVM, Cluj-Napoca, Romania. The journal allows the author(s) to hold the copyright/to retain publishing rights without restriction. 